\title{
Bacterial colonization of distal airways in healthy subjects and chronic lung disease: a bronchoscopic study
}

\author{
H. Cabello*, A. Torres*, R. Celis*, M. El-Ebiary*, J. Puig de la Bellacasa**, \\ A. Xaubet*, J. González**, C. Agustí*, N. Soler*
}

Bacterial colonization of distal airways in healthy subjects and chronic lung disease: a bronchoscopic study. H. Cabello, A. Torres, R. Celis, M. El-Ebiary, J. Puig de la Bellacasa, A. Xaubet, J. González, C. Agustí, N. Soler. @ERS Journals Ltd 1997.

ABSTRACT: In contrast to the healthy population, distal airway bacterial colonization may occur in patients with chronic lung diseases, who often have altered pulmonary defences. However, the information dealing with this issue is insufficient and is based mainly on nonspecific samples, such as sputum cultures.

Using quantitative cultures of bronchoscopic protected specimen brush (PSB) and bronchoalveolar lavage (BAL) samples, we studied the bacterial colonization of distal airways in 16 healthy subjects, 33 patients with bronchogenic carcinoma, 18 with chronic obstructive pulmonary disease (COPD), 17 with bronchiectasis, and 32 with a long-term tracheostomy due to laryngeal carcinoma. All patients were without exacerbation, and free from antibiotic treatment at least 1 month before the study protocol. Thresholds for quantitative cultures to define colonization were $\geq 10^{2}$ colony-forming units (cfu) $\mathrm{mL}^{-1}$ for $\mathrm{PSB}$ and $\geq 10^{3} \mathrm{cfu} \cdot \mathrm{mL}^{-1}$ for $B A L$.

Only one healthy subject was colonized by a potential pathogenic microorganism (PPM) (Staphylococcus aureus $4 \times 10^{2} \mathrm{cfu} \cdot \mathrm{mL}^{-1}$ in a PSB culture). Colonization was observed in $14(42 \%)$ bronchogenic carcinoma patients (19 non-PPMs, and 10 PPMs); in $15(83 \%)$ COPD patients (22 non-PPMs and 7 PPMs); in $15(88 \%)$ bronchiectasis patients (20 non-PPMs and 13 PPMs); and in $15(47 \%)$ long-term tracheostomy patients (5 non-PPMs and 13 PPMs). The two most frequent nonPPMs isolated in all groups studied were Streptococcus viridans and Neisseria spp. Haemophilus spp., Streptococcus pneumoniae, Haemophilus influenzae, and Moraxella catarrhalis were the most frequent PPMs isolated in bronchogenic carcinoma, COPD, bronchiectasis and long-term tracheostomized patients, respectively. Pseudomonas aeruginosa colonization was infrequent in all the groups.

Our results show that distal airway bacterial colonization is a frequent feature in stable patients with chronic lung diseases and also in patients with long-term tracheostomy. However, the pattern of colonization differs among groups studied. The knowledge of different colonization patterns may be important for future antibiotic prophylactic strategies and for the empirical antibiotic regimens when exacerbations occur in these patients.

Eur Respir J 1997; 10: 1137-1144.
* Servei de Pneumologia i Al.lèrgia Respiratòria and **Servei de Microbiologia, Department de Medicina, Universitat de Barcelona, Barcelona, Spain.

Correspondence: A. Torres

Servei de Pneumologia i Allèrgia Respiratòria

Hospital Clínic

Villarroel 170

08036 Barcelona

Spain

Keywords: Bacterial colonization bronchiectasis

bronchogenic carcinoma

chronic obstructive pulmonary disease

long-term tracheostomy

Received: September 231996

Accepted after revision January 191997

Grant support: Fundació Privada Clínic per a la Recerca Biomèdica/Comissió Interdepartamental per a la Recerca i Tecnologia, CIRIT (GRQ94-9103); and FIS (Fondo de Investigación Sanitaria de la Seguridad Social) $90 / 0758$ and $90 / 280$.
Distal airways are usually sterile in healthy nonsmoking individuals as has been demonstrated in various studies $[1,2]$. However, when mechanical airway defences are altered, as occurs in chronic bronchitis, chronic obstructive pulmonary disease (COPD), bronchiectasis or bronchial obstruction, or there is a bypass of oropharyngeal defences, e.g. in tracheostomized patients, the distal airways may become colonized by potential pathogenic microorganisms (PPMs) and non-potential pathogenic microorganisms (non-PPMs) [3-5]. The importance of airway bacterial colonization in these populations is not clearly understood, but it has been speculated, particularly in COPD patients, that the persistence of microorganisms in distal airways could worsen the evolution of the chronic underlying disease [6-8]. Furthermore, a knowledge of the type of colonizing agents may be important to standardize empirical antibiotic strategies when these patients develop infectious exacerbations (tracheobronchitis or pneumonia). It seems logical to assume that colonizing microbial flora will be responsible for these infections [9, 10].

Several studies have demonstrated frequent airway colonization in stable COPD and bronchiectatic patients $[1,3,4,11,12]$. The information regarding distal airway colonization in tracheostomized patients or those with bronchogenic carcinoma is scarce. Overall, few studies have used reliable bronchoscopic sampling methods, such as protected specimen brush (PSB) or bronchoalveolar lavage (BAL), to study this colonizing flora both in healthy subjects [13] and stable chronic lung disease 
patients $[14,15]$. In addition, the relationship between distal airway colonization and oropharyngeal flora has not been assessed. This prompted us to perform a prospective study, using PSB and BAL to investigate distal airway flora in healthy subjects and in patients with bronchogenic carcinoma, stable COPD, bronchiectasis, and long-term tracheostomy. The relationship between this flora and the oropharyngeal bacterial colonization was also investigated.

\section{Material and methods}

\section{Patients}

We studied 116 patients, who did not require hospital admission. These patients were divided into five categories: 1) healthy nonsmoking volunteers $(n=16) ; 2)$ patients with bronchogenic carcinoma $(n=33)$ with histological confirmation of malignancy (31 of these patients had concomitant COPD; in addition, they underwent their first bronchoscopic study at the time of inclusion into the present study); 3) patients with COPD ( $\mathrm{n}=18)$ according to previously defined criteria [16]. All COPD patients were in a stable clinical condition according to classical criteria [17]; 4) patients with bronchiectasis $(n=17)$ (who had clinical features, suggestive chest radiographic findings, and confirmatory computed tomography (CT) scan findings of bronchiectasis) [18]; 5) long-term tracheostomized patients $(n=32)$ due to total laryngectomy because of cancer of the larynx.

Patients included in the study had been free from pulmonary infections during the preceding 30 days. In addition, no patients had received antibiotic treatment during the last 4 weeks. Patients who were admitted to the hospital during the last 3 months, and those with immunosuppression (patients with acquired immune deficiency syndrome (AIDS)) or those receiving chemotherapy or corticosteroids (equivalent prednisone dose $\geq 20 \mathrm{mg} \cdot \mathrm{day}^{-1}$ ) were excluded. The mean \pm SD forced expiratory volume in one second (FEV1) was $68 \pm 19,77 \pm$ $19,73 \pm 12$ and $92 \pm 7$ percentage of predicted for bronchogenic carcinoma patients, COPD patients, bronchiectasis patients, and healthy volunteers, respectively. And, the mean \pm SD FEV1/forced vital capacity (FVC) was $65 \pm 11,65 \pm 10,72 \pm 25$ and $80 \pm 8$ percentage of predicted, for bronchogenic carcinoma patients, COPD patients, bronchiectasis patients, and healthy volunteers, respectively. COPD patients were classified as having: mild obstruction, FEV1 $65-80 \%$ pred $(n=13)$; or moderate obstruction, FEV1 $45-65 \%$ pred $(n=5)$; and none had severe obstruction, FEV1 $<45 \%$ pred. The ex-smoking condition was defined as the cessation of smoking habit at least 5 yrs previously. In patients with bronchiectasis, a confirmatory CT scan was defined as abnormally dilated bronchi near the periphery of the lung fields, failure of bronchi to taper as they left the hilar areas, or cystic dilatation of bronchi, with or without fluid levels. Long-term tracheostomy was defined as the presence of tracheostomy stoma for more than one year.

\section{Study protocol}

All patients gave informed consent to participate in the study. Pharyngeal swab samples were taken from
86 patients (10 healthy subjects, 23 bronchogenic carcinoma patients, 13 COPD patients, 11 bronchiectasis patients, and 29 long-term tracheostomized patients).

In COPD patients, the reason for performing a fibreoptic bronchoscopy was mild haemoptysis in 11 cases, suspicion of bronchiectasis in three, and chest radiographic alterations in the remaining four. In the remaining groups, bronchoscopy formed part of either the routine clinical practice in our institution (bronchogenic carcinoma and bronchiectasis), or the study protocol (healthy subjects and long-term tracheostomized patients).

Fibreoptic bronchoscopy (BF30; Olympus, New Hyde Park, NY, USA) was performed transnasally in all groups except in the long-term tracheostomized patients, where the bronchoscope was introduced through the tracheostomy stoma. Four percent nebulized lidocaine (during $15 \mathrm{~min}$ ) was used as local anaesthetic. No anaesthetics were instilled through the working channel of the bronchoscope. No aspiration was performed through the bronchoscope throughout the procedure. All bronchoscopic procedures were performed in Trendelenburg's position, to avoid oropharyngeal aspiration to the lower airways. A PSB (Microbiology brush; Mill-Rose Laboratory Inc. 7310, Mentor, Ohio, USA) sample was retrieved in all cases $(n=116)$. Samples were obtained in the right lower lobe, except in the group of patients with bronchogenic carcinoma, in whom PSB sampling was performed proximal to the endobronchial lesion, if present, or from the suspected affected bronchus corresponding to the chest radiographic infiltrate.

After PSB sampling, the fibreoptic bronchoscope was wedged into a subsegmentary bronchus from the same area where PSB was performed. BAL samples were obtained in 102 cases (15 healthy subjects, 28 bronchogenic carcinoma, 16 COPD, 13 bronchiectasis, and 30 long-term tracheostomies). Three aliquots of sterile saline $(50 \mathrm{~mL}$ each) were instilled and aspirated. The first aliquot was discarded. The mean BAL fluid obtained for processing was $27 \pm 12 \mathrm{~mL}$. The PSB procedure was always performed before BAL sampling to avoid bacterial contamination from the bronchoscope channel. For the purpose of the study, colonization of lower airways cultures was considered if PSB and BAL cultures yielded $\geq 10^{2}$ and $\geq 10^{3}$ colony-forming units (cfu) $\cdot \mathrm{mL}^{-1}$, respectively. On the other hand, cultures were considered negative if they were sterile or yielded $<10^{2}$ and $<10^{3} \mathrm{cfu} \cdot \mathrm{mL}^{-1}$ for PSB and BAL, respectively. These cut-off points were chosen arbitrarily. Bacteriological results were also evaluated using higher thresholds: $\geq 10^{3}$ $\mathrm{cfu} \cdot \mathrm{mL}^{-1}$ for PSB and $\geq 10^{4} \mathrm{cfu} \cdot \mathrm{mL}^{-1}$ for BAL.

\section{Microbiological processing}

Pharyngeal samples were obtained by means of sterile swabs (Eurotubo; Industrias Aulabor, S.A., Barcelona, Spain), homogenized in $1 \mathrm{~mL}$ of distilled water, and diluted to concentrations of $10^{-1}, 10^{-2}$ and $10^{-3}$.

The protected specimen brushes were aseptically cut into a sterile tube containing $1 \mathrm{~mL}$ of saline and agitated in a vortex-type mixer for $1 \mathrm{~min}$. Serial dilutions $\left(10^{-1}, 10^{-2}\right.$ and $\left.10^{-3}\right)$ from each PSB and BAL sample were prepared in sterile normal saline. One hundred 
microlitres of each dilution were inoculated into the following agar media: 5\% sheep blood; chocolate; Center for Disease Control (CDC); blood agar McConkey; buffered charcoal yeast extract (BCYE- $\alpha$ ); and Sabouraud dextrose. All cultures were incubated at $37^{\circ} \mathrm{C}$ under aerobic and anaerobic conditions and in a $\mathrm{CO}_{2}$-enriched atmosphere. Cultures were evaluated for growth after 24 and $48 \mathrm{~h}$ and discarded, if negative, after 5 days, with the exception of CDC blood agar which was evaluated at 7 days and for Sabouraud medium which was evaluated at 4 weeks. All microorganisms isolated were identified by standard laboratory methods [19].

Bacterial agents were classified into PPMs or nonPPMs. PPMs were those microorganisms recognized as agents causing respiratory infections, whether or not belonging to the gastrointestinal or oropharyngeal flora: Gram-negative rods, such as Pseudomonas aeruginosa, Enterobacteriaceae and Haemophilus spp.; Gram-positive cocci, such as Staphylococcus aureus, Streptococcus pneumoniae; and Gram-negative cocci, such as Moraxcella catarrhalis. Non-PPMs were those microorganisms belonging to the oropharyngeal or gastrointestinal flora that are not usually involved in respiratory infections in nonimmunocompromised patients (Streptococcus viridans group, Neisseria spp., Corynebacterium spp., Candida spp., and others) [20].

\section{Results}

A total of 116 cases was included in the study. The general characteristics of the study population are summarized in table 1 . Healthy subjects were younger than

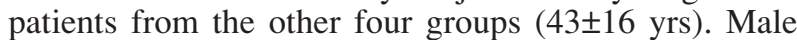
subjects were more common in the bronchogenic carcinoma group (97\%), COPD (83\%) and tracheostomy group $(91 \%)$, when compared to the healthy and bronchiectasis groups.

Table 1. - General characteristics of the study population

\begin{tabular}{lccccc}
\hline & $\begin{array}{c}\text { Healthy } \\
(\mathrm{n}=16)\end{array}$ & $\begin{array}{c}\text { Bronchogenic carcinoma } \\
(\mathrm{n}=33)\end{array}$ & $\begin{array}{c}\text { COPD } \\
(\mathrm{n}=18)\end{array}$ & $\begin{array}{c}\text { Bronchiectasis } \\
(\mathrm{n}=17)\end{array}$ & $\begin{array}{c}\text { Tracheostomy } \\
(\mathrm{n}=32)\end{array}$ \\
\hline Age yrs ${ }^{+}$ & $43 \pm 16$ & $64 \pm 10$ & $60 \pm 12$ & $57 \pm 19$ & $61 \pm 9$ \\
Gender M/F & $7 / 9$ & $32 / 1$ & $15 / 3$ & $8 / 9$ & $29 / 3$ \\
Smoking habit $\%$ & & & & & 4 \\
$\quad$ Never & 69 & 0 & 0 & 59 & 96 \\
Ex-smoker & 19 & 34 & 50 & 29 & 0 \\
Smoker & 12 & 66 & 50 & 12 & \\
\hline
\end{tabular}

COPD: chronic obstructive pulmonary disease; M: male; F: female. ${ }^{+}$: values are mean, \pm SD.

Table 2. - Number of patients with sterile or positive protected specimen brush (PSB) quantitative cultures

\begin{tabular}{lccccc}
\hline PSB cultures & $\begin{array}{c}\text { Healthy } \\
(\mathrm{n}=16)\end{array}$ & $\begin{array}{c}\text { Bronchogenic carcinoma } \\
(\mathrm{n}=33)\end{array}$ & $\begin{array}{c}\text { COPD } \\
(\mathrm{n}=18)\end{array}$ & $\begin{array}{c}\text { Bronchiectasis } \\
(\mathrm{n}=17)\end{array}$ & $\begin{array}{c}\text { Tracheostomy } \\
(\mathrm{n}=32)\end{array}$ \\
\hline Sterile & 11 & 16 & 2 & 2 & 19 \\
$<10^{2} \mathrm{cfu} \cdot \mathrm{mL}^{-1}$ & 3 & 4 & 1 & 1 & 1 \\
$10^{2}-10^{3} \mathrm{cfu} \cdot \mathrm{mL}^{-1}$ & 2 & 4 & 5 & 3 & 2 \\
$10^{3}-10^{4} \mathrm{cfu} \cdot \mathrm{mL}^{-1}$ & 0 & 5 & 6 & 3 & 3 \\
$10^{4}-10^{5} \mathrm{cfu} \cdot \mathrm{mL}^{-1}$ & 0 & 4 & 4 & 5 & 1 \\
$\geq 10^{5} \mathrm{cfu} \cdot \mathrm{mL}^{-1}$ & 0 & 0 & 0 & 3 & 2 \\
\hline
\end{tabular}

COPD: chronic obstructive pulmonary disease; cfu: colony-forming units.

Table 3. - Microorganisms isolated from PSB quantitative cultures in counts $\geq 10^{2} \mathrm{cfu} \cdot \mathrm{mL}^{-1}$

\begin{tabular}{|c|c|c|c|c|c|}
\hline & Healthy & Bronchogenic carcinoma & COPD & Bronchiectasis & Tracheostomy \\
\hline Streptococcus viridans group & $1(33)$ & $8(32)$ & $11(41)$ & $7(29)$ & $2(15)$ \\
\hline $\begin{array}{l}\text { Streptococcus group D } \\
\text { (non-Enterococcus) }\end{array}$ & $1(33)$ & - & - & $1(4)$ & - \\
\hline Streptococcus pneumoniae & - & $2(8)$ & $2(7)$ & - & $1(8)$ \\
\hline Staphylococcus aureus & $1(33)$ & $1(4)$ & $1(4)$ & $1(4)$ & $3(23)$ \\
\hline PC-negative Staphylococcus & - & $2(8)$ & $2(7)$ & $3(13)$ & - \\
\hline Micrococcus spp. & - & $1(4)$ & - & - & - \\
\hline Pseudomonas aeruginosa & - & $1(4)$ & - & $1(4)$ & - \\
\hline Moraxella catarrhalis & - & - & - & - & $4(31)$ \\
\hline Haemophilus influenzae & - & $3(12)$ & $2(7)$ & $10(42)$ & $2(15)$ \\
\hline Haemophilus parainfluenzae & - & $2(8)$ & - & - & - \\
\hline Neisseria spp. & - & $5(20)$ & $6(22)$ & $1(4)$ & - \\
\hline Corynebacterium spp. & - & - & $2(7)$ & - & $1(8)$ \\
\hline Candida spp. & - & - & $1(4)$ & - & - \\
\hline Total & 3 & 25 & 27 & 24 & 13 \\
\hline
\end{tabular}

Values are presented as absolute number of isolations, and percentage of total isolations from each group in parenthesis. PC: plasma coagulase; COPD: chronic obstructive pulmonary disease; PSB: protected specimen brush. 


\section{Colonization in healthy subjects}

PSB was performed in all $(n=16)$ healthy subjects, whilst BAL was performed in $15(94 \%)$ cases. The quantitative cultures of PSB were negative (sterile cultures or cultures $\left.<10^{2} \mathrm{cfu} \cdot \mathrm{mL}^{-1}\right)$ in $14(88 \%)$ cases. In the remaining two (12\%) cases, there were 3 microorganisms in counts $\geq 10^{2} \mathrm{cfu} \cdot \mathrm{mL}^{-1}$ in PSB samples (table 2 ). These isolates were $S$. viridans group $\left(2 \times 10^{2} \mathrm{cfu} \cdot \mathrm{mL}^{-1}\right)$, Streptococcus group $\mathrm{D}$ (non-Enterococcus) $\left(5 \times 10^{2} \mathrm{cfu} \cdot \mathrm{mL}^{-1}\right)$, and $S$. aureus $\left(4 \times 10^{2} \mathrm{cfu} \cdot \mathrm{mL}^{-1}\right)$ (table 3$)$.

BAL quantitative cultures were negative in 13 out of $15(87 \%)$ cases (sterile counts or $<10^{3} \mathrm{cfu} \cdot \mathrm{mL}^{-1}$ ) (table $4)$. In the remaining two subjects, BAL samples yielded two microorganisms: S. viridans $\left(10^{3} \mathrm{cfu} \cdot \mathrm{mL}^{-1}\right)$, and Neisseria spp. $\left(\times 10^{3} \mathrm{cfu} \cdot \mathrm{mL}^{-1}\right)($ table 5$)$.

Using PSB and BAL culture results together, four patients $(25 \%)$ were colonized, mostly by non-PPM. When cut-off points were raised to $\geq 10^{3} \mathrm{cfu} \cdot \mathrm{mL}^{-1}$ for PSB and to $\geq 10^{4} \mathrm{cfu} \cdot \mathrm{mL}^{-1}$ for BAL, none of the subjects studied demonstrated colonization (tables 2 and 4).

\section{Colonization in patients with bronchogenic carcinoma}

PSB samples were negative in 20 out of $33(61 \%)$ patients, and positive in the remaining 13 (39\%) (table 2). In the 13 colonized patients (PSB counts $\geq 10^{2} \mathrm{cfu} \cdot \mathrm{mL}^{-1}$ ), PSB samples yielded 25 microorganisms. Sixteen (64\%) of the isolated microorganisms were non-PPMs: S. viri- dans group (8); Neisseria spp. (5); plasma coagulase (PC)-negative Staphylococcus (2); and Micrococcus spp. (1). The most frequent PPMs were Haemophilus spp $(\mathrm{n}=3)$ and $S$. pneumoniae $(\mathrm{n}=2)$ (table 3$)$.

BAL quantitative cultures were negative in 24 out of $28(86 \%)$ cases (table 4$)$. In four patients, BAL samples yielded positive cultures ( $\mathrm{n}=7$ microorganisms): $S$. viridans group (3); S. aureus (1); P. aeruginosa (1); Haemophilus influenzae (1); and Neisseria spp. 1 (table 5).

When considering PSB and BAL culture results together, 14 patients (42\%) were colonized. When the cutoff points were changed to $\geq 10^{3} \mathrm{cfu} \cdot \mathrm{mL}^{-1}$ for PSB and to $\geq 10^{4} \mathrm{cfu} \cdot \mathrm{mL}^{-1}$ for BAL, nine patients $(27 \%)$ had distal airway colonization (tables 2 and 4).

\section{Colonization in patients with COPD}

PSB samples were negative in 3 out of 18 (17\%) patients, and positive in the remaining $15(83 \%)$ (table $2)$. In the 15 colonized patients, PSB samples yielded 27 microorganisms (table 3$)$. Twenty two $(81 \%)$ of the isolated microorganisms were non-PPMs: S. viridans group (11), Neisseria spp. (6); PC-negative Staphylococcus (2); Corynebacterium spp. (2); and Candida spp. (1). The PPM isolated (n=5) were: H. influenzae (2); S. pneumoniae (2); and $S$. aureus (1) (table 3).

BAL quantitative cultures were negative in 14 out of $16(88 \%)$ cases (table 4$)$. In two patients, BAL yielded

Table 4. - Number of patients with sterile or positive bronchoalveolar lavage (BAL) quantitative cultures

\begin{tabular}{lccccc}
\hline BAL cultures & $\begin{array}{c}\text { Healthy } \\
(\mathrm{n}=15)\end{array}$ & $\begin{array}{c}\text { Bronchogenic } \\
\text { carcinoma } \\
(\mathrm{n}=28)\end{array}$ & $\begin{array}{c}\text { COPD } \\
(\mathrm{n}=16)\end{array}$ & $\begin{array}{c}\text { Bronchiectasis } \\
(\mathrm{n}=13)\end{array}$ & $\begin{array}{c}\text { Tracheostomy } \\
(\mathrm{n}=30)\end{array}$ \\
\hline Sterile & 6 & 9 & 5 & 1 & 12 \\
$1-10^{2} \mathrm{cfu} \cdot \mathrm{mL}-1$ & 2 & 11 & 7 & 0 & 2 \\
$10^{2}-10^{3} \mathrm{cfu} \cdot \mathrm{mL}-1$ & 5 & 4 & 2 & 2 & 7 \\
$10^{3}-10^{4} \mathrm{cfu} \cdot \mathrm{mL}^{-1}$ & 2 & 3 & 0 & 3 & 2 \\
$10^{4}-10^{5} \mathrm{cfu} \cdot \mathrm{mL}^{-1}$ & 0 & 1 & 0 & 5 & 1 \\
$10^{5}-10^{6} \mathrm{cfu} \cdot \mathrm{mL}^{-1}$ & 0 & 0 & 0 & 1 & 0 \\
$>10^{6} \mathrm{cfu} \cdot \mathrm{mL}-1$ & 0 & 0 & 2 & 1 & 7 \\
\hline
\end{tabular}

COPD: chronic obstructive pulmonary disease; cfu: colony-forming units.

Table 5. - Microorganisms isolated from bronchoalveolar lavage (BAL) quantitative cultures

\begin{tabular}{|c|c|c|c|c|c|}
\hline & Healthy & $\begin{array}{l}\text { Bronchogenic } \\
\text { carcinoma }\end{array}$ & COPD & Bronchiectasis & Tracheostomy \\
\hline Streptococcus viridans group & $1(50)$ & $3(43)$ & $1(50)$ & $6(30)$ & - \\
\hline Streptococcus pneumoniae & - & - & $1(50)$ & - & $1(8)$ \\
\hline Staphylococcus aureus & - & $1(14)$ & - & - & $4(33)$ \\
\hline PC-negative Staphylococcus & - & - & - & - & $1(8)$ \\
\hline Pseudomonas aeruginosa & - & $1(14)$ & - & $1(5)$ & - \\
\hline Moraxella catarrhalis & - & - & - & - & $4(33)$ \\
\hline Haemophilus influenzae & - & $1(14)$ & - & $7(35)$ & - \\
\hline Neisseria spp. & $1(50)$ & $1(14)$ & - & $5(25)$ & - \\
\hline Corynebacterium spp. & $x^{2}$ & - & - & $1(5)$ & $2(17)$ \\
\hline Total & 2 & 7 & 2 & 20 & 12 \\
\hline
\end{tabular}

Values are presented as absolute number of isolations, and percentage of total isolations from each group in parenthesis. PC: plasma coagulase; COPD: chronic obstructive pulmonary disease. 
positive cultures ( $\mathrm{n}=2$ microorganisms): $S$. viridans group (1) and S. pneumoniae (1) (table 5).

The total percentage of colonization according to PSB plus BAL results was $83 \%(n=15)$. Using the cutoff point of $\geq 10^{3} \mathrm{cfu} \cdot \mathrm{mL}^{-1}$ for PSB and $\geq 10^{4} \mathrm{cfu} \cdot \mathrm{mL}^{-1}$ for BAL $56 \%$ of patients $(n=10)$ had distal airway colonization (tables 2 and 4).

\section{Colonization in patients with bronchiectasis}

PSB samples were negative in 3 out of $17(18 \%)$ patients, and positive in the remaining 14 (82\%) (table 2). In the 14 colonized patients, PSB samples yielded 24 microorganisms (table 3$)$. Twelve $(50 \%)$ of the isolated microorganisms were non-PPMs: S. viridans group (7); Neisseria spp. (1); PC-negative Staphylococcus (3); and Streptococcus group D (non-Enterococcus) (1). Among the PPMs ( $\mathrm{n}=12$ microorganisms), H. influenzae $(\mathrm{n}=10)$ was the most frequently isolated (table 3$)$.

BAL quantitative cultures were negative in 3 out of $13(23 \%)$ cases (table 4$)$. In 10 patients, BAL samples yielded 20 microbial agents, as shown in table 5 .

Taking into account both PSB and BAL culture results, distal airway colonization was observed in $88 \%$ of patients $(n=15)$. Using the cut-off point of $\geq 10^{3} \mathrm{cfu} \cdot \mathrm{mL}$ for PSB and $\geq 10^{4} \mathrm{cfu} \cdot \mathrm{mL}^{-1}$ for BAL $64 \%$ of patients $(n=11)$ had distal airway colonization (tables 2 and 4$)$.

\section{Colonization in long-term tracheostomized patients}

PSB samples were negative in 20 out of $32(62 \%)$ patients, and positive in the remaining 12 (38\%) (table $2)$. In the 12 colonized patients, PSB samples yielded 13 microorganisms (table 3). Three (23\%) of the isolated microorganisms were non-PPMs: $S$. viridans group (2); and Corynebacterium spp. (1). The predominant PPM $(\mathrm{n}=10)$ were: $M$. catarrhalis (4) and Staphylococcus aureus (3) (table 3 ).

BAL quantitative cultures were negative in 20 out of $30(67 \%)$ cases (table 4$)$. In 10 patients, BAL samples yielded 12 microbial agents as shown in table 5 .

The total percentage of colonization (PSB plus BAL cultures) was $47 \%(n=15)$. When using higher cut-off points for PSB $\left(\geq 10^{3} \mathrm{cfu} \cdot \mathrm{mL}^{-1}\right)$ and BAL $\left(\geq 10^{4} \mathrm{cfu} \cdot \mathrm{mL}^{-1}\right)$ the total percentage of distal airway colonization was $28 \%(n=9)$ (tables 2 and 4$)$.
Microbial isolates from PSB $\left(<10^{2} \mathrm{cfu} \cdot \mathrm{mL}^{-1}\right)$ and $B A L$ samples $\left(<10^{3} \mathrm{cfu} \cdot \mathrm{mL}^{-1}\right)$

In healthy subjects, three cases yielded $S$. viridans from their PSB cultures in counts $<10^{2} \mathrm{cfu} \cdot \mathrm{mL}^{-1}$. The following microorganisms were isolated from BAL cultures of healthy subjects in counts $<10^{3} \mathrm{cfu} \cdot \mathrm{mL}^{-1}$ in seven cases. S. viridans (4); Streptococcus group D (1); PC-negative Staphylococcus (1); and S. viridans + PCnegative Staphylococcus + Neisseria spp. (1).

In the group of patients with bronchogenic carcinoma, the following microorganisms were isolated from PSB cultures in counts $<10^{2} \mathrm{cfu} \cdot \mathrm{mL}^{-1}$ in three patients: S. pneumoniae (1); PC-positive Staphylococcus (1); Neisseria spp. (1). The following microorganisms were isolated from BAL cultures in counts $<10^{3} \mathrm{cfu} \cdot \mathrm{mL}^{-1}$ in 15 cases: S. viridans (6); PC-negative Staphylococcus (1); PC-positive Staphylococcus (1); S. pneumoniae (1); $H$. influenzae (1); S. viridans + PC-negative Staphylococcus (1); S. viridans + PC-positive Staphylococcus (1); Neisseria spp. (1); S. viridans + Neisseria spp. (1); and Enterobacter sakazakii (1).

In COPD patients, only one case yielded $S$. viridans from PSB cultures $<10^{2} \mathrm{cfu} \cdot \mathrm{mL}^{-1}$. As regards BAL cultures $<10^{3} \mathrm{cfu} \cdot \mathrm{mL}^{-1}$, nine patients yielded the following microorganisms: S. viridans + Neisseria spp. (3); S. viridans (2); Neisseria spp. (2); S. pneumoniae (1); and PCnegative Staphylococcus (1).

As regards patients with bronchiectasis, only one case yielded $S$. viridans from PSB cultures $<10^{2} \mathrm{cfu} \cdot \mathrm{mL}^{-1}$. From BAL cultures $\left(<10^{3} \mathrm{cfu} \cdot \mathrm{mL}^{-1}\right)$, two patients yielded S. pneumoniae + PC-negative Staphylococcus, and the other yielded $P$. aeruginosa + Neisseria spp.

Finally, patients with long-term tracheostomies yielded Neisseria spp. from PSB cultures in counts $<10^{2}$ $\mathrm{cfu} \cdot \mathrm{mL}^{-1}$ in only one case. BAL cultures $<10^{3} \mathrm{cfu} \cdot \mathrm{mL}^{-1}$ from eight patients yielded: PC-positive Staphylococcus (2); Neisseria spp. (2); S. pneumoniae (1); Streptococcus group G (1); S. viridans + PC-positive Staphylococcus + Corynebacterium spp. (1); M. catarrhalis (1).

\section{Qualitative agreement between pharyngeal swab, PSB,} and BAL isolate

Pharyngeal swabs were taken from 86 patients yielding a total of 217 microorganisms. In all these patients PSB and BAL samples were also obtained. The results of the qualitative concordance between pharyngeal swabs

Table 6. - Qualitative concordance between microbial species isolated from pharyngeal swabs, protected specimen brush (PSB) samples and bronchoalveolar lavage (BAL) samples

\begin{tabular}{|c|c|c|c|c|c|c|c|}
\hline \multirow[t]{3}{*}{ Group } & \multirow{3}{*}{$\begin{array}{l}\mathrm{PhS} \\
\mathrm{n} *\end{array}$} & \multirow{2}{*}{\multicolumn{3}{|c|}{$\begin{array}{l}\text { PSB } \\
\text { Coincident } \\
\text { organisms with PhS }\end{array}$}} & \multirow{2}{*}{\multicolumn{3}{|c|}{$\begin{array}{c}\text { BAL } \\
\text { Coincident } \\
\text { microorganisms with } \mathrm{PhS}\end{array}$}} \\
\hline & & & & & & & \\
\hline & & $\mathrm{n}^{*}$ & PPMs & Non-PPMs & $\mathrm{n}^{*}$ & PPMs & Non-PPMs \\
\hline Healthy subjects $(n=10)$ & 33 & 5 & 0 & $4(80)$ & 11 & 0 & $10(91)$ \\
\hline Bronchogenic carcinoma $(n=23)$ & 62 & 27 & 0 & $12(44)$ & 20 & 0 & $13(65)$ \\
\hline COPD $(n=13)$ & 30 & 19 & 0 & $11(58)$ & 11 & 0 & $7(64)$ \\
\hline Bronchiectasis $(n=110)$ & 29 & 15 & 0 & $5(33)$ & 24 & $3(12)$ & $10(42)$ \\
\hline Tracheostomy $(n=29)$ & 63 & 16 & $2(12)$ & $5(31)$ & 28 & $2(7)$ & $8(28)$ \\
\hline
\end{tabular}

*: number of isolates. The values in parenthesis are percentages. PPMs: potential pathogenic microorganisms; non-PPMs: nonpotential pathogenic microorganisms; PhS: pharyngeal swabs; COPD: chronic obstructive pulmonary disease. 
and PSB and BAL cultures are presented in table 6. The percentage of concordance was higher in healthy subjects $(80 \%$ for PSB and $91 \%$ for BAL) compared to the remaining groups (table 6). Non-PPMs accounted for most qualitative concordances among the three types of cultures (pharyngeal swab, PSB and BAL).

\section{Discussion}

The main finding of the present study was that distal airways are frequently colonized in clinically stable populations with bronchogenic carcinoma (42\%), COPD $(83 \%)$, bronchiectasis $(88 \%)$ and in long-term tracheostomized patients (47\%). Healthy subjects were insignificantly colonized. The most frequent microorganisms found in each group were non-PPMs, such as S. viridans group and Neisseria spp., except for tracheostomized patients, in whom PPMs (M. catarrhalis and S. aureus) were the most frequent colonizers. These results reveal the possible pathogens related to bacterial exacerbations in the populations studied.

Healthy nonsmokers are free from bacterial colonization of the lower airways. Nevertheless, there is very little information dealing with this issue [13]. In the present study, we found that only one healthy subject had a true respiratory pathogen isolated in significant numbers $\left(4 \times 10^{2} \mathrm{cfu} \cdot \mathrm{mL}^{-1} \mathrm{~S}\right.$. aureus $)$ from his lower airways. KIRKPATRICK and BASS [13], using PSB and BAL to study the distal airways of eight healthy people, found that only one BAL specimen yielded one PPM. Similar results were also reported in a recently published article [14]. The present study and the latter two underline the efficacy of lung defences in healthy subjects in maintaining the near-sterility of the lower airways.

For several years, it has been believed that COPD patients had their distal airways colonized by bacteria $[3,4,12]$. In addition, an increase in the airway bacterial burden was a constant feature during exacerbations [15]. However, this general conviction comes from studies that have used diagnostic methods with high risk of oropharyngeal microbial contamination, such as sputum cultures [3]. A recent article from Monso et al. [15], using fibreoptic PSB samples, demonstratéd that $25 \%$ of 40 stable COPD patients had colonization of the distal airways (cut-off $\geq 10^{3} \mathrm{cfu} \cdot \mathrm{mL}^{-1}$ ), although they did not distinguish potential pathogenic from nonpotential pathogenic microorganisms. Similar results were reported by RIISE et al. [14] studying 18 COPD patients. The present results in the COPD group, although using a lower threshold, demonstrated a higher rate of colonization $(83 \%)$. However, $76 \%$ of the colonizing agents were non-PPMs belonging to the oropharyngeal flora (tables 3 and 5). We do not know the significance of the presence of these non-PPMs in the lower airways. The measurement of inflammatory cytokine production by the airways could clarify the meaning of both types of microorganisms (PPMs and non-PPMs) colonizing the distal airways of stable COPD patients. More studies are still warranted to confirm the frequently and importance of non-PPM colonizing microorganisms in COPD patients. Additionally, it could help to explain the "vicious circle" hypothesis regarding the role of bacterial infection in COPD [21].

The PPMs (S. pneumoniae, H. influenzae and S. aureus) found in the present study represented $24 \%$ of the total number of colonizing agents corresponding to seven COPD patients. We did not observe Gram-negative bacilli, such as $P$. aeruginosa, which is in agreement with other studies in the literature $[1-4,11,14]$. However, we do not know whether more severely ill COPD patients, needing antibiotics frequently and/or receiving longterm corticosteroids, could have enteric Gram-negative bacilli. Enteric Gram-negative bacilli were also found infrequently during COPD exacerbations requiring or not requiring hospitalization $[15,22]$. Nonetheless, in COPD exacerbated patients requiring mechanical ventilation, $P$. aeruginosa, Proteus mirabilis and Escherichia coli accounted for $18 \%$ of the total microorganisms recovered [23], suggesting a relationship between the severity of the exacerbation and the presence of these microorganisms. In addition, this latter finding may have important implications for antibiotic therapy. A possible limitation of the present study as regards COPD patients could be the bias due to studying this population because of the distinct clinical indications. However, the majority of patients were investigated because of mild haemoptysis. Moreover, all patients were free from exacerbation.

Results of the study of colonization pattern in patients with bronchogenic carcinoma did not differ from those found in COPD patients, indicating that both populations were very similar as demonstrated by the results of forced spirometry. However, one case of $P$. aeruginosa was found in the bronchogenic carcinoma group. Another study of patients with bronchogenic carcinoma found similar PSB isolates as in the present study, and yet the authors studied the contralateral lung [24]. Overall, the presence of bronchogenic carcinoma does not seem to modify the colonizing bacterial flora in COPD patients. However, others have shown that Gramnegative bacilli and anaerobes may be found in distal airways below the bronchial obstruction [25]. We believe that a knowledge of bacterial colonization in patients with bronchogenic carcinoma may be important for the development of prophylactic strategies, especially when they are undergoing thoracic surgery.

The most frequent PPM found in distal airways from patients with bronchiectasis was $H$. influenzae. This finding coincides with most series dealing with bronchoscopic and nonbronchoscopic sampling in bronchiectatic populations [6, 26-28]; and indicates that the spectrum of empirical antibiotic treatment in bronchiectasis has to cover this microorganism. P. aeruginosa was extremely infrequent in the present series (one case; $<6 \%$ ). This figure is significantly low compared to the $10 \%$ incidence $[6,28]$, or to the $24-31 \%$ incidence $[26$, 29] reported by other authors. Explanations for discrepancies among studies regarding $P$. aeruginosa in bronchiectasis are based on the different diagnostic methods used, and also on difference in severity of airway obstruction. For instance, Evans et al. [6] confirmed that bronchiectatic patients colonized by $P$. aeruginosa had lower FEV1 (28\% pred) when compared to those who did not yield this microorganism (59\% pred). Therefore, empirical coverage against $P$. aeruginosa is mandatory in exacerbated patients with bronchiectasis and severe obstruction. In fact, a large study on severe community-acquired pneumonia in which only five patients had 
$P$. aeruginosa, revealed that all five had bronchiectasis [30].

We also investigated tracheostomized nonhospitalized patients. Surprisingly, $M$. catarrhalis accounted for the majority of PPMs, followed by S. aureus. There is a disparity between the bacterial flora found in this group and that found in the remaining groups, indicating that long-term tracheostomized patients constitute a peculiar group in terms of distal airway colonization. Unfortunately, we do not know how many patients from this group had COPD. This is a significant finding for the empirical antibiotic strategy when these patients develop tracheobronchitis or pneumonia. Moreover, there is very little information regarding airway colonization in nonhospitalized long-term tracheostomized patients. A recent paper from HARLID et al. [31] demonstrated a 30\% colonization of the distal airways in this type of patient. The predominant microorganisms isolated were $S$. aureus, enteric Gram-negative bacilli, and $P$. aeruginosa. As regards the latter microorganism, there is clear evidence that tracheostomized patients (particularly post- Intensive Care Unit (ICU)) have a predisposition to become colonized by $P$. aeruginosa [32-34]. The colonization probably starts in the stoma and migrates down the trachea, where microorganisms have a peculiar affinity for epithelial adherence [35, 36]. We found a discrepancy between pharyngeal and distal colonization in these patients, thus, we have to assume that the stoma is the introductory airway colonization gate, as has been suggested by other authors [31]. Unfortunately, we only have stoma swab cultures in few patients from this group.

Overall, the percentage of positive cultures was much lower in BAL compared to PSB samples except in patients with bronchiectasis. These results clearly suggest that bacterial colonization is located mainly at the bronchial level. However, in bronchiectasis it seems to be a persistent bacterial burden in alveolar spaces. These patients could have more substantial alterations in their lung defences compared to the remaining populations studied.

A potential criticism of the present study may be the choice of low thresholds for quantitative cultures of PSB $\left(\geq 10^{2} \mathrm{cfu} \cdot \mathrm{mL}^{-1}\right)$ and BAL $\left(\geq 10^{3} \mathrm{cfu} \cdot \mathrm{mL}^{-1}\right)$. In fact, other studies $[14,15]$ have used higher thresholds $\left(\geq 10^{3}\right.$ $\mathrm{cfu} \cdot \mathrm{mL}^{-1}$ for PSB). Since the latter cut-off point for PSB was originally [37] defined to distinguish colonization from infection, and the present study was designed to study colonization of distal airways, we thought that using lower thresholds would avoid missing important microbiological information regarding colonization. Conversely, we could have chosen lower thresholds to differentiate colonization from infection, consequently increasing the rate of colonization, particularly with BAL. However, we have ignored the meaning of microorganisms isolated in very low counts (PSB $<10^{2}$ $\mathrm{cfu} \cdot \mathrm{mL}^{-1}$ and $\left.\mathrm{BAL}<10^{3} \mathrm{cfu} \cdot \mathrm{mL}^{-1}\right)$.

It is commonly believed that prior abnormal oropharyngeal colonization is the main mechanism for subsequent distal airway colonization [38]. In healthy subjects, $80 \%$ of the microorganisms found in distal airways were also isolated from the oropharynx (table 6). However, the percentage of qualitative agreement between both sites decreased to around 50\% in other patient groups, and was even lower in patients with long-term tracheostomy, in agreement with a previous study [39]. Discrepancies between pharyngeal and distal airway cultures were even higher for $H$. influenzae in pati-ents with bronchiectasis. The lack of concordance between pharyngeal swabs and PSB or BAL cultures could be explained as follows: 1) pharyngeal swabs may not be the best samples since they can miss bacteriological information; some authors recommend pharyngeal gargles [40]; 2) pharyngeal colonization may, perhaps, be a transient feature, whilst distal airway colonization is a constant one; 3) other oropharyngeal-related reservoirs for distal airway colonization that were not evaluated in the present study could play a role, e.g., sinuses [41] and dental plaque [42]; and, finally, 4) some microorganisms, such as $P$. aeruginosa and $H$. influenzae have special affinity due to their ability of adhere to tracheal cells without previous oropharyngeal colonization, particularly in specific populations, such as patients with COPD or bronchiectasis [31, 34].

In summary, our results confirm that colonization of distal airways is a frequent finding in stable patients with chronic lung disease or long-term tracheostomy. Our findings reinforce the idea that populations with alterations in their lung defences are chronically colonized. The clinical importance of this chronic colonization warrants future research.

\section{References}

1. Lees AW, McNaught W. Bacteriology of the lower respiratory tract secretions: sputum and upper respiratory tract secretions in "normals" and chronic bronchitis. Lancet 1959; ii: 1112-1115.

2. Laurenzi GA, Potter RT, Kass EH. Bacteriologic flora of the lower respiratory tract. Lancet 1961; 265: 12731278 .

3. Brumfitt W, Willoughby M, Bromley L. Evaluation of sputum in chronic bronchitis. Lancet 1957; 2: 13061309.

4. Potter RT, Rotman F, Fernández F, et al. The bacteriology of the lower respiratory tract. Am Rev Respir Dis 1967; 97: 1051-1061.

5. Brook Y. Bacterial colonization, tracheobronchitis, and pneumonia following tracheostomy and long-term intubation in pediatric patients. Chest 1979; 76: 420-424.

6. Evans SA, Turner SM, Bosch BJ, et al. The influence of chronic colonization with Pseudomonas aeruginosa on lung function in bronchiectasis. Eur Respir J 1996; 9: 1601-1604.

7. Nagaki M, Shimura S, Tanno Y, et al. Role of Pseudomonas aeruginosa infection in the development of bronchiectasis. Chest 1992; 102: 1464-1469.

8. Murphy TF, Sethi S. Bacterial infection in chronic obstructive pulmonary disease. Am Rev Respir Dis 1992; 146: 1067-1083.

9. Fagon JY, Chastre J. Severe exacerbations of COPD patients: the role of pulmonary infections. Semin Respir Infect 1996; 11: 109-118.

10. Ferguson G, Cherniack R. Management of chronic obstructive lung disease. N Engl J Med 1993; 328: 10171022.

11. Hass H, Morris J, Samson S, et al. Bacterial flora of the respiratory tract in chronic bronchitis: comparison of transtracheal, fiberbronchoscopic and oropharyngeal sampling methods. Am Rev Respir Dis 1977; 116: 41-47. 
12. Gump DW, Phillips A, Forsyth R, et al. Role of infection in chronic bronchitis. Am Rev Respir Dis 1976; 113: 465-474.

13. Kirkpatrick MB, Bass JB. Quantitative bacterial cultures of bronchoalveolar lavage fluids and protected specimens from normal subjects. Am Rev Respir Dis 1989; 139: 546-548.

14. Riise GC, Larsson S, Larsson P, et al. The intrabronchial microbial flora in chronic bronchitis patients: a target for N-acetylcysteine therapy? Eur Respir J 1994 7: 94-101.

15. Monsó E, Ruiz J, Rosell A, et al. Bacterial infection in chronic obstructive pulmonary disease: A study of stable and exacerbated out-patients using the protected specimen brush. Am J Respir Crit Care Med 1995; 152: $1316-1320$

16. American Thoracic Society. Standards for the diagnosis and care of patients with chronic obstructive pulmonary disease. Am J Respir Crit Care Med 1995; 152: S77-S120.

17. Anthonisen NR, Manfreda J, Warren CPW, et al. Antibiotic therapy in exacerbations of chronic obstructive lung disease. Ann Intern Med 1987; 106: 196-204.

18. Nicotra MB. Bronchiectasis. Semin Respir Infect 1994; 9: 31-40.

19. Ballows A, Harsler WJ Jr. In: Manual of Clinical Microbiology. 5th Edn. Section III. Washington, DC, American Society for Microbiology, 1991; pp. 209-553.

20. Murray A, Mostafa S, van Saene H. Essentials in clinical microbiology. In: Soutenbeek CP, van Saene HKF, eds. "Baillieres's: Clinical Anaesthesiology" 1991; 5: pp. 1-23.

21. Cole P. Host-microbe relationships in chronic respiratory infections. Respiration 1989; 55 (Suppl. 1): 5 8.

22. Martínez JA, Rodríguez E, Bastida T, et al. Quantitative study of the bronchial bacterial flora in acute exacerbations of chronic bronchitis. Chest 1994; 105: 976.

23. Fagon JY, Chastre J, Trouillet JL, et al. Characterization of distal bronchial microflora during acute exacerbation of chronic bronchitis. Am Rev Respir Dis 1990; 142: 1004-1008.

24. Vereen L, Smart L, George R. Antibody coating and quantitative cultures of bacteria in sputum and bronchial brush specimens from patients with stable chronic bronchitis. Chest 1986; 90: 534-536.

25. Yuang-Shuang L, Pan-Chyr Y, Zeg-Guang W, et al. The bacteriology of obstructive pneumonitis. Am J Respir Crit Care Med 1994; 149: 1648-1653.

26. Pang J, Cheng A, Chan HS, et al. The bacteriology of bronchiectasis in Hong Kong investigated by protected specimen brush and bronchoalveolar lavage. Am Rev Respir Dis 1989; 139: 14-17.

27. Bjerkestrand G, Digranes A, Schreiner A. Bacteriological findings in transtracheal aspirates from patients with chronic bronchitis and bronchiectasis. Scand J Respir Dis 1975; 56: 201-207.

28. Hill SL, Burnett D, Hewetson A, et al. The response of patients with purulent bronchiectasis to antibiotics for four months. $Q J$ Med 1988; 250: 163-173.

29. Nicotra MB, Rivera M, Dale A, et al. Clinical, pathophysiologic and microbiologic characterization of bronchiectasis in an aging cohort. Chest 1995; 108: 955-961.

30. Torres A, Serra-Batlles J, Ferrer A, et al. Severe community-acquired pneumonia. Am Rev Respir Dis 1991; 144: 312-318.

31. Harlid R, Andersson G, Claes GF, et al. Respiratory tract colonization in patients with chronic tracheostomy. Am J Respir Crit Care Med 1996; 154: 124-129.

32. Tano Y, Matsushima T, Kobashi Y, et al. Bacterial flora of the respiratory tract in patients with long-term tracheostomy: colonization of the lower respiratory tract by Pseudomonas aeruginosa. Nippon Kyobu Shikkan Gakkai Zachi 1994; 32: 146-151.

33. Niederman MS, Ferranti RD, Zeigler A, et al. Respiratory infection complicating long-term tracheostomy: the implication of persistent gram-negative tracheobronchial colonization. Chest 1984; 85: 39-44.

34. Niederman MS, Mantovani R, Schoch P, et al. Patterns and routes of tracheobronchial colonization in mechanically-ventilated patients: the role of nutritional status in colonization of lower airway by Pseudomonas species. Chest 1989; 95: 155-161.

35. Niederman MS, Rafferty TD, Sasaki CT, et al. Comparison of bacterial adherence to ciliated and squamous epithelial cells obtained from the human respiratory tract. Am Rev Respir Dis 1983; 127: 85-90.

36. Palmer L, Donelan S, Fox G, et al. Gastric flora in chronically mechanically-ventilated patients: relationship to upper and lower airway colonization. Am J Respir Crit Care Med 1995; 151: 1063-1067.

37. Wimberley N, Bass J, Boyd BW, et al. Use of a bronchoscopic protected catheter brush for the diagnosis of pulmonary infections. Chest 1982; 81: 556-562.

38. Johanson W, Pierce A, Sanford J, et al. Nosocomial respiratory infections with Gram-negative bacilli. Ann Intern Med 1972; 77: 701-706.

39. Bartlett J, Faling J, Willey S. Quantitative tracheal bacteriologic and cytologic studies in patients with longterm tracheostomies. Chest 1978; 74: 635-639.

40. Van Saene HK, Stoutenbeek CP, Torres A. The abnormal oropharyngeal carrier state: symptom or disease? Respir Med 1992; 86: 183-186.

41. Rouby JJ, Laurent P, Gosnach M, et al. Risk factors and clinical relevance of nosocomial maxillary sinusitis in the critically ill. Am J Respir Crit Care Med 1994; 150: 776-783.

42. Scannapieco FA, Stewart E, Mylotte J. Colonization of dental plaque by respiratory pathogens in medical intensive care patients. Crit Care Med 1992; 20: 740-745. 